\title{
SOBOLEV-LIKE HILBERT SPACES INDUCED BY ELLIPTIC OPERATORS
}

\author{
TETIANA KASIRENKO, VLADIMIR MIKHAILETS, AND ALEKSANDR MURACH
}

\begin{abstract}
We investigate properties of function spaces induced by the inner product Sobolev spaces $H^{s}(\Omega)$ over a bounded Euclidean domain $\Omega$ and by an elliptic differential operator $A$ on $\bar{\Omega}$. The domain and the coefficients of $A$ are of the class $C^{\infty}$. These spaces consist of all distributions $u \in H^{s}(\Omega)$ such that $A u \in H^{\lambda}(\Omega)$ and are endowed with the corresponding graph norm, with $s, \lambda \in \mathbb{R}$. We prove an interpolation formula for these spaces and discuss their application to elliptic boundary-value problems.
\end{abstract}

\section{INTRODUCTION}

This paper is devoted to the function spaces induced by the scale $\left\{H^{s}(\Omega): s \in \mathbb{R}\right\}$ of inner product Sobolev spaces over a bounded Euclidean domain $\Omega$ and by an elliptic partial differential operator $A$ on $\bar{\Omega}$. The boundary of $\Omega$ and the coefficients of $A$ are supposed to be infinitely smooth. We investigate the inner product spaces $H_{A, \lambda}^{s}(\Omega)$, where $s, \lambda \in \mathbb{R}$, that consist of all distributions $u \in H^{s}(\Omega)$ subject to the condition $A u \in H^{\lambda}(\Omega)$ and that are endowed with the corresponding graph norm. We call $H_{A, \lambda}^{s}(\Omega)$ the Sobolev-like space induced by $A$. Such spaces are used in the theory of elliptic differential operators and elliptic boundary-value problems.

Specifically, the space $H_{A, 0}^{0}(\Omega)$ is the domain of the maximal operator that corresponds to the unbounded operator $A$ defined on $C^{\infty}(\bar{\Omega})$ and acting in $L_{2}(\Omega)[1,2,3]$. Lions and Magenes $[4,5]$ systematically use the space $H_{A, 0}^{s}(\Omega)$ for $s<\operatorname{ord} A=: 2 q$ in the theory of solvability of regular elliptic boundary-value problems in distribution spaces. In the case of the Dirichlet problem, the space $H_{A,-q}^{s}(\Omega)$ for $s<q$ is also useful [6]. These spaces serve as domains of Fredholm bounded operators that correspond to elliptic problems. A more general class of the spaces $H_{A, \lambda}^{s}(\Omega)$ is considered in $[7,8]$. Some of their versions are introduced for $L_{p}$-Sobolev spaces $[4,5,9,10]$, weighted Sobolev spaces [10, 11, 12], and generalized Sobolev spaces $[8,13,14]$ and are also used in the theory of elliptic differential equations.

The purpose of this paper is to investigate some properties of the spaces $H_{A, \lambda}^{s}(\Omega)$ concerning their completeness, separability, and dense subsets. Besides, we will prove an interpolation formula for these spaces. We also give their application to elliptic boundary-value problems.

2010 Mathematics Subject Classification. 46E35, 35J30, 35J40.

Key words and phrases. Sobolev space, elliptic operator, complex interpolation, elliptic problem, Fredholm operator. 


\section{Main Results}

Let $1 \leq n \in \mathbb{Z}$ and $s \in \mathbb{R}$. We let $H^{s}\left(\mathbb{R}^{n}\right)$ denote the complex inner product Sobolev space of order $s$ over $\mathbb{R}^{n}$. By definition, this space consists of all tempered distributions $w$ in $\mathbb{R}^{n}$ that their Fourier transform $\widehat{w}$ is locally Lebesgue integrable over $\mathbb{R}^{n}$ and satisfies the condition

$$
\|w\|_{s, \mathbb{R}^{n}}^{2}:=\int_{\mathbb{R}^{n}}\left(1+|\xi|^{2}\right)^{s}|\widehat{w}(\xi)|^{2} d \xi<\infty .
$$

The space $H^{s}\left(\mathbb{R}^{n}\right)$ is Hilbert and separable with respect to the norm $\|\cdot\|_{s, \mathbb{R}^{n}}$. If $\Omega$ is an open subset of $\mathbb{R}^{n}$, then the Sobolev space $H^{s}(\Omega)$ of order $s$ over $\Omega$ is defined to consist of the restrictions $u:=w\left\lceil\Omega\right.$ of all distributions $w \in H^{s}\left(\mathbb{R}^{n}\right)$ to $\Omega$. This space is Hilbert and separable with respect to the norm

$$
\|u\|_{s, \Omega}:=\inf \left\{\|w\|_{s, \mathbb{R}^{n}}: w \in H^{s}\left(\mathbb{R}^{n}\right), u=w\lceil\Omega\}\right.
$$

and is continuously embedded in the linear topological space $\mathcal{D}^{\prime}(\Omega)$ of all distributions in $\Omega$.

Henceforth we suppose that $n \geq 2$ and that $\Omega$ is a bounded domain whose boundary $\Gamma:=\partial \Omega$ is an infinitely smooth closed manifold of dimension $n-1$, the $C^{\infty}$ structure on $\Gamma$ being induced by $\mathbb{R}^{n}$. In the closed domain $\bar{\Omega}:=\Omega \cup \Gamma$, we consider an arbitrary elliptic linear partial differential expression

$$
A:=\sum_{|\mu| \leq 2 q} a_{\mu}(x) \frac{\partial^{|\mu|}}{\partial_{x_{1}}^{\mu_{1}} \cdots \partial_{x_{n}}^{\mu_{n}}}
$$

of an even order $2 q \geq 2$ with complex-valued coefficients $a_{\mu} \in C^{\infty}(\bar{\Omega})$. Here, $\mu:=\left(\mu_{1}, \ldots, \mu_{n}\right)$ is a multi-index with nonnegative integer-valued components, $|\mu|:=\mu_{1}+\cdots+\mu_{n}$, and $x=\left(x_{1}, \ldots, x_{n}\right)$ is an arbitrary point in $\mathbb{R}^{n}$. The ellipticity condition for $A$ means that

$$
\sum_{|\mu|=2 q} a_{\mu}(x) \xi_{1}^{\mu_{1}} \cdots \xi_{n}^{\mu_{n}} \neq 0
$$

for every point $x \in \bar{\Omega}$ and each vector $\xi=\left(\xi_{1}, \ldots, \xi_{n}\right) \in \mathbb{R}^{n} \backslash\{0\}$. In the $n=2$ case, we suppose in addition that $A$ is properly elliptic on $\bar{\Omega}$ (see, e.g., [15, Section 5.2.1]).

We associate some spaces with the elliptic formal differential operator $A$. Choosing $s, \lambda \in \mathbb{R}$ arbitrarily, we let $H_{A, \lambda}^{s}(\Omega)$ denote the linear space

$$
\left\{u \in H^{s}(\Omega): A u \in H^{\lambda}(\Omega)\right\}
$$

endowed with the graph norm

$$
\|u\|_{s, A, \lambda}:=\left(\|u\|_{s, \Omega}^{2}+\|A u\|_{\lambda, \Omega}^{2}\right)^{1 / 2} .
$$

Here and below, $A u$ is understood in the sense of the theory of distributions in $\Omega$.

Theorem 1. Let $s, \lambda \in \mathbb{R}$. The following assertions are true:

(i) The space $H_{A, \lambda}^{s}(\Omega)$ is Hilbert and separable. 
(ii) The equality of linear spaces $H_{A, \lambda}^{s}(\Omega)$ and $H^{s}(\Omega)$ holds if and only if $\lambda \leq$ $s-2 q$. If $\lambda \leq s-2 q$, the norms in these spaces are equivalent.

(iii) The set $C^{\infty}(\bar{\Omega})$ is dense in $H_{A, \lambda}^{s}(\Omega)$.

(iv) If $s \leq 1 / 2$ and $\lambda \leq 1 / 2-2 q$, then the set $C_{0}^{\infty}(\Omega)$ is dense in $H_{A, \lambda}^{s}(\Omega)$.

Here and below, $C_{0}^{\infty}(\Omega)$ denotes the set of all functions from $C^{\infty}(\bar{\Omega})$ that vanish near the boundary of $\Omega$.

Note that, if $\lambda>s-2 q$, the space $H_{A, \lambda}^{s}(\Omega)$ depends on the coefficients of $A$, even when all of them are constant. For example, if $A_{1}$ and $A_{2}$ are elliptic constantcoefficient linear differential expressions, the embedding $H_{A_{1}, 0}^{0}(\Omega) \subseteq H_{A_{2}, 0}^{0}(\Omega)$ implies that $A_{2}=\alpha A_{1}+\beta$ for certain $\alpha, \beta \in \mathbb{C}$. This follows from Hörmander's result [2, Theorem 3.1].

It is well known that the Sobolev scale $\left\{H^{s}(\Omega): s \in \mathbb{R}\right\}$ has the following interpolation property: if $s_{0}, s_{1} \in \mathbb{R}$ and $0<\theta<1$, then

$$
\left[H^{s_{0}}(\Omega), H^{s_{1}}(\Omega)\right]_{\theta}=H^{(1-\theta) s_{0}+\theta s_{1}}(\Omega)
$$

up to equivalence of norms (see, e.g., [15, Section 4.3.1, Theorem 1]). Here and below, $\left[H_{0}, H_{1}\right]_{\theta}$ denotes the result of the complex interpolation with the parameter $\theta$ of a compatible pair of Hilbert (or, more generally, Banach) spaces $H_{0}$ and $H_{1}$ (see, e.g., [15, Section 1.9]). The pair $\left[H_{0}, H_{1}\right]$ of these spaces is called compatible if they are continuously embedded in a certain linear Hausdorff space.

Let us formulate a version of the interpolation property (1) for the spaces $H_{A, \lambda_{j}}^{s_{j}}(\Omega)$, where $j \in\{0,1\}$. In view of assertion (ii) of Theorem 1 , it is worthwhile to restrict ourselves to the case when $\lambda_{j} \geq s_{j}-2 q$.

Theorem 2. Suppose that $s_{0}, s_{1}, \lambda_{0}, \lambda_{1}$, and $\theta$ are real numbers satisfying the inequalities $\lambda_{0} \geq s_{0}-2 q, \lambda_{1} \geq s_{1}-2 q$, and $0<\theta<1$. Put $s:=(1-\theta) s_{0}+\theta s_{1}$ and $\lambda:=(1-\theta) \lambda_{0}+\theta \lambda_{1}$. Then

$$
\left[H_{A, \lambda_{0}}^{s_{0}}(\Omega), H_{A, \lambda_{1}}^{s_{1}}(\Omega)\right]_{\theta}=H_{A, \lambda}^{s}(\Omega)
$$

up to equivalence of norms.

\section{Proof of the MAIN RESUlts}

We will give a joint proof of Theorems 1 and 2 .

Let us first prove that the space $H_{A, \lambda}^{s}(\Omega)$ is Hilbert for arbitrary $s, \lambda \in \mathbb{R}$. Evidently, the norm in it is induced by a graph inner product. Besides, the space $H_{A, \lambda}^{s}(\Omega)$ is complete. Indeed, if $\left(u_{k}\right)$ is a Cauchy sequence in this space, then there exist the limits $u:=\lim u_{k}$ in $H^{s}(\Omega)$ and $f:=\lim A u_{k}$ in $H^{\lambda}(\Omega)$ because the last two spaces are complete. The differential operator $A$ is continuous on $\mathcal{D}^{\prime}(\Omega)$; therefore, $A u=\lim A u_{k}=f$ in $\mathcal{D}^{\prime}(\Omega)$. Here, $u \in H^{s}(\Omega)$ and $f \in H^{\lambda}(\Omega)$. Hence, $u \in H_{A, \lambda}^{s}(\Omega)$, and $\lim u_{k}=u$ in the space $H_{A, \lambda}^{s}(\Omega)$. Thus, this space is complete.

Let us now prove assertion (ii) of Theorem 1 . Since ord $A=2 q$, the differential operator $A$ acts continuously from $H^{s}(\Omega)$ to $H^{s-2 q}(\Omega)$ for every $s \in \mathbb{R}$. Hence, if 
$\lambda \leq s-2 q$, then $H^{s}(\Omega)=H_{A, \lambda}^{s}(\Omega)$ up to equivalence of norms due to the continuous embedding $H^{s-2 q}(\Omega) \subseteq H^{\lambda}(\Omega)$.

Conversely, assume that $H^{s}(\Omega)=H_{A, \lambda}^{s}(\Omega)$ for certain $s, \lambda \in \mathbb{R}$. Choose an arbitrary distribution $w \in H^{s}\left(\mathbb{R}^{n}\right)$ such that $\operatorname{supp} w \subset \Omega$, and put $u:=w \uparrow \Omega$. Since $u \in H^{s}(\Omega)$, we have the inclusion $A u \in H^{\lambda}(\Omega)$ by our assumption. Hence, $\chi u \in H^{\lambda+2 q}(\Omega)$ for every function $\chi \in C_{0}^{\infty}(\Omega)$ by virtue of the ellipticity of $A$ (see, e.g., [16, Theorem 7.4.1]). We can choose the function $\chi$ so that $\chi=1$ on $\operatorname{supp} w$. Therefore $u \in H^{\lambda+2 q}(\Omega)$, which implies the inclusion $w \in H^{\lambda+2 q}\left(\mathbb{R}^{n}\right)$. Thus,

$$
\left\{w \in H^{s}\left(\mathbb{R}^{n}\right): \operatorname{supp} w \subset \Omega\right\} \subseteq H^{\lambda+2 q}\left(\mathbb{R}^{n}\right) .
$$

According to [16, Theorem 2.2.2], this embedding implies that

$$
(1+|\xi|)^{\lambda+2 q} \leq c(1+|\xi|)^{s}
$$

for every $\xi \in \mathbb{R}^{n}$ with some number $c>0$ not depending on $\xi$. Hence, $\lambda+2 q \leq s$. We have proved assertion (ii) of Theorem 1.

To prove Theorem 2 and the remaining part of Theorem 1, we use a theorem on interpolation of some Hilbert spaces induced by linear continuous operators. Before we formulate this result, let us make some notation. If $H, \Phi$ and $\Psi$ are Hilbert spaces subject to the continuous embedding $\Phi \subseteq \Psi$ and if $T: H \rightarrow \Psi$ is a continuous linear operator, we put

$$
(H)_{T, \Phi}:=\{u \in H: T u \in \Phi\}
$$

and endow the linear space $(H)_{T, \Phi}$ with the graph norm

$$
\|u\|_{(H)_{T, \Phi}}:=\left(\|u\|_{H}^{2}+\|T u\|_{\Phi}^{2}\right)^{1 / 2} .
$$

This norm does not depend on $\Psi$, and the space $(H)_{T, \Phi}$ is Hilbert. The latter is proved in a quite similar way as the proof of the completeness of $H_{A, \lambda}^{s}(\Omega)$.

Proposition 1. Assume that six separable Hilbert spaces $X_{0}, Y_{0}, Z_{0}, X_{1}, Y_{1}$, and $Z_{1}$ and three linear mappings $T, R$, and $S$ are given and satisfy the following conditions:

(i) The pairs $\left[X_{0}, X_{1}\right]$ and $\left[Y_{0}, Y_{1}\right]$ are compatible.

(ii) The spaces $Z_{0}$ and $Z_{1}$ are linear manifolds in a certain locally convex topological space $E$.

(iii) The continuous embeddings $Y_{0} \subseteq Z_{0}$ and $Y_{1} \subseteq Z_{1}$ hold.

(iv) The mapping $T$ is given on $X_{0}+X_{1}$ and defines the bounded operators $T: X_{0} \rightarrow Z_{0}$ and $T: X_{1} \rightarrow Z_{1}$.

(v) The mapping $R$ is given on $E$ and defines the bounded operators $R: Z_{0} \rightarrow X_{0}$ and $R: Z_{1} \rightarrow X_{1}$.

(vi) The mapping $S$ is given on $E$ and defines the bounded operators $S: Z_{0} \rightarrow Y_{0}$ and $S: Z_{1} \rightarrow Y_{1}$.

(vii) The equality $T R u=u+S u$ holds for every $u \in E$.

Then

$$
\left[\left(X_{0}\right)_{T, Y_{0}},\left(X_{1}\right)_{T, Y_{1}}\right]_{\theta}=\left(\left[X_{0}, X_{1}\right]_{\theta}\right)_{T,\left[Y_{0}, Y_{1}\right]_{\theta}}
$$


up to equivalence of norms for every $\theta \in(0,1)$.

This result is proved by Lions and Magenes [12, Chapter 1, Theorem 14.3] in a more general case of Banach spaces.

Let us now prove Theorem 2. Our reasoning is motivated by [12, Chapter 2, Proof of Theorem 4.2] and [8, Section 3.3.4]. We choose an integer $r \geq 1$ arbitrarily and consider the linear differential expression $A^{r} A^{r+}+1$ of order $4 q r$. Here, as usual, $A^{r+}$ denotes the differential expression which is formally adjoint to the $r$-th iteration $A^{r}$ of $A$. Given an integer $\sigma \geq 2 q r$, we let $H_{D}^{\sigma}(\Omega)$ denote the linear manifold of all distributions $u \in H^{\sigma}(\Omega)$ such that $\partial_{\nu}^{j} u=0$ on $\Gamma$ for each $j \in\{0, \ldots, 2 q r-1\}$. Here, $\partial_{\nu}$ is the operator of the differentiation with respect to the inward normal to the boundary $\Gamma$ of $\Omega$. This definition is reasonable in view of the theorem on traces of distributions from $H^{\sigma}(\Omega)$ (see, e.g., [15, Section 4.7.1]). According to this theorem, $H_{D}^{\sigma}(\Omega)$ is a (closed) subspace of $H^{\sigma}(\Omega)$. We have the isomorphism

$$
A^{r} A^{r+}+1: H_{D}^{\sigma}(\Omega) \leftrightarrow H^{\sigma-4 q r}(\Omega)
$$

for every integer $\sigma \geq 2 q r$ (see, e.g., [8, Lemma 3.1]). Let $\left(A^{r} A^{r+}+1\right)^{-1}$ denote the inverse of this isomorphism. This inverse defines the continuous linear operator

$$
\left(A^{r} A^{r+}+1\right)^{-1}: H^{l}(\Omega) \rightarrow H^{l+4 q r}(\Omega)
$$

for every integer $l \geq-2 q r$. It follows from the interpolation formula (1) that this operator is well defined and continuous for every real $l \geq-2 q r$.

In Proposition 1 , we put $X_{j}:=H^{s_{j}}(\Omega), Y_{j}:=H^{\lambda_{j}}(\Omega), Z_{j}:=H^{s_{j}-2 q}(\Omega), E:=$ $H^{\min \left\{s_{0}, s_{1}\right\}-2 q}(\Omega)$, and $T:=A$. Evidently, conditions (i)-(iv) of this proposition are fulfilled. Besides, subjecting $r$ to the restrictions

$$
s_{j}-2 q \geq-2 q r \quad \text { and } \quad s_{j}-2 q-\lambda_{j} \geq-4 q r
$$

for each $j \in\{0,1\}$, we put $R:=A^{r-1} A^{r+}\left(A^{r} A^{r+}+1\right)^{-1}$ and $S:=-\left(A^{r} A^{r+}+1\right)^{-1}$. Owing to (4), we have the continuous linear operators

$$
R: Z_{j}=H^{s_{j}-2 q}(\Omega) \rightarrow H^{s_{j}}(\Omega)=X_{j}
$$

and

$$
S: Z_{j}=H^{s_{j}-2 q}(\Omega) \rightarrow H^{s_{j}-2 q+4 q r}(\Omega) \subseteq H^{\lambda_{j}}(\Omega)=Y_{j}
$$

for each $j \in\{0,1\}$ (the last embedding is continuous); i.e., conditions (v) and (vi) are fulfilled as well. The last condition (vii) is satisfied because

$$
T R u=\left(A^{r} A^{r+}+1-1\right)\left(A^{r} A^{r+}+1\right)^{-1} u=u+S u
$$

for every $u \in E$. Thus, owing to Proposition 1 and the interpolation formula (1), we conclude that

$$
\begin{aligned}
{\left[H_{A, \lambda_{0}}^{s_{0}}(\Omega), H_{A, \lambda_{1}}^{s_{1}}(\Omega)\right]_{\theta} } & =\left[\left(X_{0}\right)_{T, Y_{0}},\left(X_{1}\right)_{T, Y_{1}}\right]_{\theta} \\
& =\left(\left[X_{0}, X_{1}\right]_{\theta}\right)_{T,\left[Y_{0}, Y_{1}\right]_{\theta}}=H_{A, \lambda}^{s}(\Omega)
\end{aligned}
$$

up to equivalence of norms, which proves Theorem 2.

Let us prove the remaining part of Theorem 1. We make use of the following supplement to Proposition 1: 
Proposition 2. Suppose that the hypothesis of Proposition 1 is fulfilled. If the Hilbert spaces $X_{0}, Y_{0}, X_{1}$, and $Y_{1}$ are separable and satisfy the dense continuous embeddings $X_{1} \subseteq X_{0}$ and $Y_{1} \subseteq Y_{0}$, then the Hilbert spaces $\left(X_{0}\right)_{T, Y_{0}}$ and $\left(X_{1}\right)_{T, Y_{1}}$ are also separable and satisfy the dense continuous embedding $\left(X_{1}\right)_{T, Y_{1}} \subseteq\left(X_{0}\right)_{T, Y_{0}}$.

This result is proved in [17, Theorem 4.1] for a more general method of interpolation between Hilbert spaces than that used in the paper (see also [8, Section 3.3.2]).

According to Proposition 2, the spaces $H_{A, \lambda_{0}}^{s_{0}}(\Omega)$ and $H_{A, \lambda_{1}}^{s_{1}}(\Omega)$ from Theorem 2 are separable, and the second of them is continuously and densely embedded in the first provided that $s_{0} \leq s_{1}$ and $\lambda_{0} \leq \lambda_{1}$. This specifically implies assertion (i) of Theorem 1 concerning the separability of $H_{A, \lambda}^{s}(\Omega)$ for all $s \in \mathbb{R}$ and $\lambda>s-2 q$. If $\lambda \leq s-2 q$, this separability follows from assertion (ii).

It remains to prove assertions (iii) and (iv) of Theorem 1 . Let $s, \lambda \in \mathbb{R}$; if $\lambda>s-2 q$, we have the dense continuous embedding

$$
H^{s_{1}}(\Omega)=H_{A, s_{1}-2 q}^{s_{1}}(\Omega) \subseteq H_{A, \lambda}^{s}(\Omega)
$$

for every number $s_{1} \in \mathbb{R}$ such that $s_{1} \geq s$ and $s_{1}-2 q \geq \lambda$. This has been noted in the previous paragraph. Since the set $C^{\infty}(\bar{\Omega})$ is dense in $H^{s_{1}}(\Omega)$, this embedding implies assertion (iii) in the case of $\lambda>s-2 q$. In the opposite case, this assertion is a consequence of assertion (ii).

Finally, assume that $s \leq 1 / 2$ and $\lambda \leq 1 / 2-2 q$. If $\lambda>s-2 q$, assertion (iv) follows from (5) and the density of $C_{0}^{\infty}(\Omega)$ in $H^{s_{1}}(\Omega)$ for $s_{1} \leq 1 / 2$ (see, e.g., [15, Section 4.3.2, Theorem 1 (a)]). If $\lambda \leq s-2 q$, this assertion follows from this density in view of assertion (ii). Our proof is complete.

\section{Application}

Let us discuss an application of the space $H_{A, \lambda}^{s}(\Omega)$ to elliptic boundary-value problems. We consider a boundary-value problem that consists of the elliptic equation

$$
A u=f \quad \text { in } \quad \Omega
$$

and the boundary conditions

$$
B_{j} u=g_{j} \quad \text { on } \quad \Gamma, \quad j=1, \ldots, q .
$$

Here, each

$$
B_{j}:=\sum_{|\mu| \leq m_{j}} b_{j, \mu}(x) \frac{\partial^{|\mu|}}{\partial_{x_{1}}^{\mu_{1}} \cdots \partial_{x_{n}}^{\mu_{n}}}
$$

is a linear boundary partial differential expression on $\Gamma$ of order $m_{j} \leq 2 q-1$ with complex-valued coefficients $b_{j, \mu} \in C^{\infty}(\Gamma)$. We suppose that the boundary-value problem (6), (7) is elliptic in $\Omega$ (see the definition in, e.g., [19, Section 1.2]). Put $m:=\max \left\{m_{1}, \ldots, m_{q}\right\}$; the case of $m \geq 2 q$ is possible.

It is known [18, Theorem 7] that the mapping

$$
u \mapsto(A u, B u)=\left(A u, B_{1} u, \ldots, B_{q} u\right),
$$


where $u \in C^{\infty}(\bar{\Omega})$, extends uniquely (by continuity) to a Fredholm continuous linear operator

$$
(A, B): H^{s}(\Omega) \rightarrow H^{s-2 q}(\Omega) \oplus \bigoplus_{j=1}^{q} H^{s-m_{j}-1 / 2}(\Gamma)
$$

for every real number $s>m+1 / 2$. Moreover, the kernel of this operator lies in $C^{\infty}(\bar{\Omega})$ and together with the index of the operator does not depend on $s$. Here and below, $H^{\sigma}(\Gamma)$ denotes the inner product Sobolev space over $\Gamma$ of order $\sigma \in \mathbb{R}$. Let $N$ and $\varkappa$ respectively denote these kernel and index.

This fundamental property of problem (6), (7) does not remain for $s \leq m+1 / 2$. Specifically, if $s \leq m_{j}+1 / 2$, then the mapping $u \mapsto B_{j} u$, where $u \in C^{\infty}(\bar{\Omega})$, cannot be extended to a continuous linear operator from $H^{s}(\Omega)$ to the linear topological space $\mathcal{D}^{\prime}(\Gamma)$ of all distributions on $\Gamma$. But it is possible to prove a version of this property for every $s \leq m+1 / 2$ provided that we use some spaces $H_{A, \lambda}^{s}(\Omega)$ instead of $H^{s}(\Omega)$.

Theorem 3. Let numbers $s, \lambda \in \mathbb{R}$ satisfy the conditions $s \leq m+1 / 2, \lambda>-1 / 2$, and $\lambda>m+1 / 2-2 q$. Then the mapping (8), where $u \in C^{\infty}(\bar{\Omega})$, extends uniquely (by continuity) to a Fredholm continuous linear operator

$$
(A, B): H_{A, \lambda}^{s}(\Omega) \rightarrow H^{\lambda}(\Omega) \oplus \bigoplus_{j=1}^{q} H^{s-m_{j}-1 / 2}(\Gamma) .
$$

The kernel of this operator equals $N$, and the index equals $\varkappa$.

If $s \in \mathbb{Z}$ and $s \leq 0$, Theorem 3 is proved in a somewhat similar way as that used in [7] and [8, Sections 4.4.2 and 4.4.3] for regular elliptic boundary-value problems. (The corresponding reasoning is given in [20, Section 5] for $m \geq 2 q$ ). In the general situation we prove Theorem 3 with the help of the interpolation formula (2). Choose $l \in \mathbb{Z}$ such that $l<s$ and $l \leq 0$. We consider the Fredholm continuous operators

$$
(A, B): H_{A, \lambda}^{l}(\Omega) \rightarrow H^{\lambda}(\Omega) \oplus \bigoplus_{j=1}^{q} H^{l-m_{j}-1 / 2}(\Gamma)
$$

and

$$
(A, B): H^{\lambda+2 q}(\Omega) \rightarrow H^{\lambda}(\Omega) \oplus \bigoplus_{j=1}^{q} H^{\lambda+2 q-m_{j}-1 / 2}(\Gamma)
$$

(note that $\lambda+2 q>1 / 2$ by the hypothesis of the Theorem). They have the common kernel $N$ and the common index $\varkappa$. Since $l<s<\lambda+2 q$, there exists a number $\theta \in(0,1)$ such that $s=(1-\theta) l+\theta(\lambda+2 q)$. Applying the interpolation with the parameter $\theta$ to these operators, we conclude by [8, Theorem 1.5] that a restriction of the first operator is a Fredholm continuous operator between the spaces

$$
\left[H_{A, \lambda}^{l}(\Omega), H^{\lambda+2 q}(\Omega)\right]_{\theta}
$$


and

$$
\left[H^{\lambda}(\Omega) \oplus \bigoplus_{j=1}^{q} H^{l-m_{j}-1 / 2}(\Gamma), H^{\lambda}(\Omega) \oplus \bigoplus_{j=1}^{q} H^{\lambda+2 q-m_{j}-1 / 2}(\Gamma)\right]_{\theta} .
$$

Moreover, this operator has the same kernel and index as operators (10) and (11). Owing to Theorem 2, the first space coincides with $H_{A, \lambda}^{s}(\Omega)$ up to equivalence of norms (remark that $H^{\lambda+2 q}(\Omega)=H_{A, \lambda}^{\lambda+2 q}(\Omega)$ ). Besides, the second space coincides up to equivalence of norms with the target space of operator (9) due to an analog of the interpolation formula (1) for Sobolev spaces over $\Gamma$ (see, e.g, [12, Capter 1, Theorem 7.7]). Thus, the latter Fredholm operator acts between the spaces indicated in (9). It is an extension by continuity of the mapping (8), where $u \in C^{\infty}(\bar{\Omega})$, in view of assertion (iii) of Theorem 1. The proof of Theorem 3 is complete.

Remark that, in the important case of regular elliptic boundary-value problems, Theorem 3 is proved by Lions and Magenes [4, 5] in the framework of $L_{p}$-Sobolev spaces provided that $\lambda=0$ and $s \notin\{1 / p-k: 1 \leq k \in \mathbb{Z}\}$. In the case where $s \geq 0$ and $m \leq 2 q-1$, this theorem is contained in the result formulated in [19, p. 86].

\section{REFERENCES}

[1] O. A. Ladyzenskaya, On the closure of the elliptic operator (Russian), Doklady Akad. Nauk SSSR (N.S.) 79, (1951). 723-725.

[2] L. Hörmander, On the theory of general partial differential equations, Acta Math. 94 (1955), no. $1,161-248$.

[3] M. Š. Birman, Characterization of elliptic differential operators with the maximal domain of definition (Russian), Vestnik Leningrad. Univ. Ser. Math. Mech. Astr. 19 (1957), no. 4, $177-183$.

[4] J.-L. Lions, E. Magenes, Problémes aux limites non homogénes, V, Ann. Sci. Norm. Sup. Pisa 16 (1962), 1-44.

[5] J.-L. Lions, E. Magenes, Problémes aux limites non homogénes, VI, J. d'Analyse Math. 11 (1963), 165-188.

[6] J.-L. Lions, E. Magenes, Problémes aux limites non homogénes, II, Ann. Inst. Fourier (Grenoble) 11 (1961), 137-178.

[7] Murach A. A., Extension of some Lions-Magenes theorems, Methods Funct. Anal. Topology, 15 (2009), no. 2, 152-167.

[8] V. A. Mikhailets, A. A. Murach, Hörmander spaces, interpolation, and elliptic problems, de Gruyter Stud. Math., vol. 60, De Gruyter, Berlin, 2014.

[9] G. Geymonat, Sul problema di Dirichlet per le equazoni lineari ellittiche, Ann. Sci. Norm. Sup. Pisa 16 (1962), 225-284.

[10] E. Magenes, Spazi di interpolazione ed equazioni a derivate parziali, Atti VII Congr. Un. Mat. Italiana (Genoa, 1963), Edizioni Cremonese, Rome, 1965, 134-197.

[11] J.-L. Lions, E. Magenes, Problémes aux limites non homogénes, VII, Ann. Mat. Pura Appl. (4) 63 (1963), 201-224.

[12] J.-L. Lions and E. Magenes, Non-Homogeneous Boundary-Value Problems and Applications, vol. I, Grundlehren Math. Wiss., vol. 181, Springer-Verlag, New York-Heidelberg, 1972.

[13] V. A. Mikhailets and A. A. Murach, Elliptic problems and Hörmander spaces, Modern analysis and applications. The Mark Krein Centenary Conference. Vol. 2: Differential operators and mechanics, 447-470, Oper. Theory Adv. Appl. 191, Birkhäser, Basel, 2009. 
[14] V. A. Mikhailets, A. A. Murach, The refined Sobolev scale, interpolation, and elliptic problems, Banach J. Math. Anal. 6 (2012), no. 2, 211-281.

[15] H. Triebel, Interpolation Theory, Function Spaces, Differential Operators [2-nd edn], Johann Ambrosius Barth, Heidelberg, 1995.

[16] L. Hörmander, Linear Partial Differential Operators, Grundlehren Math. Wiss., vol. 116, Springer, Berlin, 1963.

[17] V. A. Mikhailets, A. A. Murach, A regular elliptic boundary-value problem for a homogeneous equation in a two-sided refined scale of spaces, Ukrainian Math. J. 58 (2006), no. 11, 17481767.

[18] J. Peetre, Another approach to elliptiv boundary-value problems, Comm. Pure Appl. Math. 14 (1961), no. 4, 711-731.

[19] M. S. Agranovich, Elliptic boundary problems, Partial differential equations, IX, 1-144, Encyclopaedia Math. Sci., 79, Springer, Berlin, 1997.

[20] A. V. Anop, T. M. Kasirenko, A. A. Murach, Nonregular elliptic boundary-value problems and Hörmander spaces, Ukraïn. Mat. Zh. 70 (2018), no. 3, 299-317.

Institute of Mathematics, National Academy of Sciences of Ukraine, 3 TERESHChENKIVS'KA, KYIV, 01601, UkRAINE

E-mail address: kasirenko@imath.kiev.ua

Institute of Mathematics, National Academy of Sciences of Ukraine, 3 TERESHChenkivs'Ka, Kyiv, 01601, Ukraine

E-mail address: mikhailets@imath.kiev.ua

Institute of Mathematics, National Academy of Sciences of Ukraine, 3 TereshChenkivs'Ka, Kyiv, 01601, Ukraine

E-mail address: murach@imath.kiev.ua 